\title{
How to restrain Auger recombination predominance in the threshold of asymmetric bi-quantum-well lasers
}

\author{
I.A. Sukhoivanov $^{\mathrm{a}, *}$, O.V. Mashoshyna ${ }^{\mathrm{b}}$, V.K. Kononenko ${ }^{\mathrm{c}}$, D.V. Ushakov ${ }^{\mathrm{c}}$

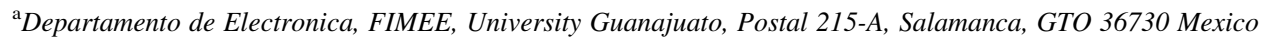 \\ ${ }^{\mathrm{b}}$ Kharkov National University of Radio Electronics, Lenin Av., 14, 61166 Kharkov, Ukraine \\ ${ }^{\mathrm{c}}$ Stepanov Institute of Physics NASB, Fr. Scorina Pr., 70, 220072 Minsk, Belarus
}

Available online 14 March 2005

\begin{abstract}
Both radiative and nonradiative processes which occur in the active region of GaInAs-GaInAsP-InP asymmetric multiple quantum-well (AMQW) heterolasers with two quantum wells of different width (4 and $9 \mathrm{~nm}$ ) are described. Several possible processes of non-radiative Auger recombination which affect the temperature sensitivity of the lasing threshold are analyzed and the temperature dependencies of the investigated processes are presented. For the above-mentioned AMQW heterostructure, it is shown that the influence of the Auger recombination processes on the temperature behaviour of the lasing threshold can be restrained by operation at temperatures lower than $340 \mathrm{~K}$ and the cavity losses which do not exceed $60 \mathrm{~cm}^{-1}$.
\end{abstract}

(C) 2005 Elsevier Ltd. All rights reserved.

PACS: 42.55.Px; 85.35.Be; 72.20.Jv; 79.20.Fv

Keywords: Auger recombination; Asymmetric quantum-well laser; Lasing threshold; Temperature parameters

\section{Introduction}

Nowadays, AMQW lasers are investigated intensively because of their wide usage. For instance, they can serve as broadband light sources and amplifiers for all optical network systems [1] and are basic components of modern telecommunication systems operating at the wavelength $1.55 \mu \mathrm{m}$. The laser diodes must be stable and have low values of the threshold current. However, at the operation temperature enhancement these devices suffer from nonradiative losses, mainly because of Auger recombination (AR) processes accelerating [2,3]. Moreover, including into consideration the processes of non-radiative AR allows to take into account the non-equilibrium processes which occur in the active region of the QW lasers more completely and accurately. Therefore, the development of long-wavelength lasers is issue of the day because the problem of the weakening of

\footnotetext{
* Corresponding author.

E-mail addresses: i.sukhoivanov@ieee.org (I.A. Sukhoivanov), mashoshina@kture.kharkov.ua (O.V. Mashoshyna), lavik@dragon.bas-
} net.by (V.K. Kononenko), ushakovdv@bsu.by (D.V. Ushakov).

0026-2692/\$ - see front matter (C) 2005 Elsevier Ltd. All rights reserved. doi:10.1016/j.mejo.2005.02.022 such processes still exists. A plausible way for solving this problem can be application of semiconductor lasers based on asymmetric quantum-well (QW) heterostructures [4].

The calculations of AMQW lasers characteristics are a complicated problem by itself and one of the main task of the problem is to carry out such calculations and another one is to include all possible processes in a theoretical model as well as describe their physics concerning with the laser operation. Thus, in this paper, we present a theoretical model which is capable of describing both spontaneous radiative recombination and AR and, as a consequence, allows to propose some operating regimes of the laser which provide the restriction of AR predominance. The results of temperature dependencies calculation of the AR rate, quantum yield of spontaneous recombination and threshold current density as well as its loss dependence in the GaInAs-GaInAsP-InP asymmetric bi-QW heterolasers with different widths of the QWs (4 and $9 \mathrm{~nm}$ ) are shown and discussed.

\section{Approach for lasing threshold}

The level of excitation at which the lasing occurs is characterized with the threshold quantity of the spontaneous 
radiative recombination rate $R_{\mathrm{sp}}$, thus in the general case the threshold current in QW heterostructure lasers $j_{\text {th }}$ is determined as

$j_{\mathrm{th}}=\frac{e d}{\eta^{\prime} \eta_{\mathrm{sp}}} R_{\mathrm{sp}}$

where $d$ is the QW width, $\eta^{\prime}$ is the injection efficiency, $\eta_{\mathrm{sp}}$ is the quantum yield of spontaneous emission. The coefficient $\eta^{\prime}$ describes the escape of injected current carriers to emitters and depends on the laser structure design, doping, and temperature. The quantity of $\eta_{\mathrm{sp}}$ characterizes the relative role of useless recombination processes in the active region, such as transitions through different defects and nonradiative AR. Increasing the temperature influences on the threshold value of the current carrier concentration that has, accordingly, an effect on $\eta^{\prime}$ and $\eta_{\mathrm{sp}}$ and on $R_{\mathrm{sp}}$ as well. In asymmetric QW heterostructures, the additional complex behaviour appears due to electron-optical interaction of the QWs in the conditions of non-uniform excitation of the active region [4]. In this case, tunneling and carrier transport through modified barriers between the QWs provide specific changes in the threshold versus temperature [4]. Below, conditions of uniform excitation of the varied width QWs are only examined.

The violation of conservation of the electron wave vector at optical transitions provides a second power dependence of $j_{\mathrm{th}} \sim T^{2}$ [5]. Involving highlying excited subbands at the transitions increases power index of the dependence $j_{\mathrm{th}}(T)$ and therefore functionally $j_{\mathrm{th}} \sim T^{n}$, where $n$ lies in the range of 1-3. The temperature decreasing of $\eta^{\prime}$ and $\eta_{\mathrm{sp}}$ has obviously a definite effect too. Additional behaviour of the lasing threshold is introduced certainly by temperature changes in the cavity losses.

Often, the temperature dependence of the threshold is described by an exponential function with the characteristic parameter $T_{0}$. However, though it is a convenient empirical parameter, its value is ordinary indefinite, depends on the temperature operation interval, and widely varies for the same laser diode type. According to the determining of $T_{0}$ one has at a narrow temperature operation interval $T \approx T_{\mathrm{op}}$ approximately $T_{0} \approx T_{\mathrm{op}} / n$.

To exclude the AR rate, we present the threshold in the form

$j_{\mathrm{th}}=\frac{e}{\eta^{\prime}}\left(A n_{\mathrm{th}}^{n}+C n_{\mathrm{th}}^{3}\right)$

where $n_{\mathrm{th}}$ is the threshold sheet concentration of electrons. The coefficient $A$ is determined by the optical transition probability and for direct dipole transitions $(n=1)$ it can be approximately taken as the Einstein coefficient $A_{\mathrm{cv}}$, i.e. in this case, $A \approx A_{\mathrm{cv}} \approx 1 / \tau_{\mathrm{sp}}$, where $\tau_{\mathrm{sp}}$ is the threshold lifetime of current carriers at spontaneous radiative recombination [6]. In the limit case of no $k$-selection rule $(n=2)$, the coefficient $A$ is also related to $A_{\mathrm{cv}}$ by a definite relation [5].
The last term in Eq. (2) $C n_{\text {th }}^{3}$ describes the AR rate $R$ since it is roughly proportional to $n_{\text {th }}^{3}$ [2]. As a rule, the AR coefficient $C$ is determined in the activation approach via a formula $C \approx C_{0} \exp \left(-E_{\text {act }} / k T\right)$ [2], where $E_{\text {act }} \approx E_{\mathrm{q}} \delta_{\mathrm{m}}, \delta_{\mathrm{m}}$ is the effective mass relation that is different for each of the AR processes, $E_{\mathrm{q}}$ is the energy of transitions between corresponding subband levels.

It is well known that for an ideal QW laser $n_{\mathrm{th}} \sim T$ [7-9] and in order to insert factors which describe nonlinearity (for our case it is AR) we expressed two threshold components from (2) with their temperature approximations that let us obtained the formula for characteristic temperature as

$T_{0} \approx \frac{T_{\mathrm{op}}}{n+\left(1-\eta_{\mathrm{sp}}\right)\left(3-n+E_{\mathrm{act}} / k T_{\mathrm{op}}\right)}$.

As seen, if the role of the AR becomes essential $\left(\eta_{\mathrm{sp}} \ll 1\right)$, the relation between the activation energy $E_{\text {act }}$ and the thermal energy $k T_{\mathrm{op}}$ determines the value of the lasing threshold characteristic parameter $T_{0}$. It is expected that in the investigated lasers AR will intensify the lasing threshold and at the same time reduce characteristic temperature values.

In the work, the temperature dependence of the lasing threshold in the GaInAs-GaInAsP-InP bi-QW heterolasers with different widths of the QWs (4 and $9 \mathrm{~nm}$ ) has been determined. Including into consideration the processes of non-radiative AR allows to take into account the nonequilibrium processes which occur in the active region of the QW lasers more completely and accurately.

\section{Investigated structure}

Numerical calculations have been performed for asymmetric bi-QW heterostructure lasers in the GaInAsGaInAsP-InP system. The active region includes two QWs of widths $d_{1}=4 \mathrm{~nm}$ and $d_{2}=9 \mathrm{~nm}$ and a barrier of the width $d_{\mathrm{b}}=15 \mathrm{~nm}$.

Energy levels in the QWs have been preliminarily determined in the effective mass approximation using standard parameters [10] for semiconductor components (Table 1). In the QW of the 4-nm width, only one subband of electrons and light holes and two subbands of heavy holes are realized, in the QW of the 9-nm width the number of possible electron and light hole subbands is one more and the number of heavy hole subbands becomes up to four. The laser diodes operate at the wavelength near $1.55 \mu \mathrm{m}$ and provide a widen tunable gain spectrum [11].

More detailed energy band structure in the QWs was calculated according to the four-band $\mathbf{k} \cdot \mathbf{p}$ method [12]. The $\mathbf{k} \cdot \mathbf{p}$ calculation results are in good agreement with the evaluations of the subband energies in the effective mass approximation and were used for determining the gain and spontaneous radiative recombination spectra of the lasers. 
Table 1

Parameters for the calculations in the effective mass approximation, $T=$ $300 \mathrm{~K}$

\begin{tabular}{lllll}
\hline Energy gap $E_{\mathrm{g}}, \mathrm{eV}$ & & \multicolumn{2}{l}{ Band offset, eV } \\
${$\cline { 1 - 1 }$} }$ & Barrier & & $\Delta E_{\mathrm{c}}$ & $\Delta E_{\mathrm{v}}$ \\
\hline 0.718 & 1.060 & 0.137 & 0.205
\end{tabular}

Effective mass in units $m_{\mathrm{e}}$

\begin{tabular}{llllll}
\hline Electrons & \multicolumn{3}{l}{ Heavy holes } & & \multicolumn{2}{l}{ Light holes } \\
\cline { 2 - 3 } \cline { 5 - 6 }$m_{\mathrm{c}}$ & $m_{\mathrm{vh}}$ & $m_{\mathrm{vht}}$ & & $m_{\mathrm{vl}}$ & $m_{\mathrm{vlt}}$ \\
\hline 0.043 & 0.307 & 0.051 & & 0.040 & 0.116 \\
\hline
\end{tabular}

Both gain spectrum and spontaneous radiative recombination spectrum have been calculated in a similar way through Lorentzian spectral broadening of the line emission [11]. At the calculation of the gain, the squared matrix element of dipole transitions is determined for a definite TEor TM-polarization while for the spontaneous recombination the matrix element of the transitions is taken as for the isotropic radiation.

The value of the optical confinement factor was evaluated in the equivalent three-layer waveguide model. Using the parameters of the QW heterostructure (Table 1), we have for the TE-mode optical confinement factors $\Gamma_{1}=$ $3.5 \times 10^{-3}$ and $\Gamma_{2}=7.9 \times 10^{-3}$ [11]. Though the optical localization of the electromagnetic wave is better in the 9-nm width QW, the value of the gain coefficient is more higher in the 4-nm width QW.

\section{Auger recombination rate: analytic expression and behaviour}

Calculations of the AR rate in the laser active region have been made according to the conventional method [2]. The next interactions were considered, i.e. ( $\mathrm{CCCH})$ three electrons and one heavy hole, (CHHL) one electron, two heavy holes and one light hole, (CHHS) one electron, two heavy holes and one split-off-band hole, and (CLLS) one electron, two light holes and one split-off-band hole. First three processes are ordinary ones which take into account at the analysis. It was found that CLLS process also contributes to AR increasing as our calculation revealed. The cause for it is relatively little value of the split-off separation energy $0.361 \mathrm{eV}$ in comparison with energy gap for the investigated structure (Table 1) which makes transitions from light-hole subband to split-off band energy-preferable.

In the analytic representation, the coefficient $C_{0}$ for considered AR processes can be written accordingly [2] in the form [9]

$C_{0}=\frac{4 \mathrm{e}^{4}\left|V_{\mathrm{T}}\right|^{2} \alpha}{\hbar k T \varepsilon^{2}}$,

where $\varepsilon$ is the dielectric constant of the semiconductor crystal, $\mathbf{V}_{\mathrm{T}}$ is the matrix element of the carrier interactions at
Table 2

The matrix element for different AR processes in the QWs at $T=300 \mathrm{~K}$

\begin{tabular}{lll}
\hline$d, \mathrm{~nm}$ & AR process & $\mathrm{cm}^{2}, \mathrm{~cm}^{-2}$ \\
\hline 4 & CCCH & $1.3 \times 10^{-13}$ \\
& CHHS & $6.3 \times 10^{-40}$ \\
& CHHL & $5.0 \times 10^{-65}$ \\
9 & CLLS & $5.5 \times 10^{-14}$ \\
& CCCH & $2.0 \times 10^{-14}$ \\
& CHHS & $2.4 \times 10^{-36}$ \\
& CHHL & $3.0 \times 10^{-59}$ \\
& CLLS & $6.2 \times 10^{-14}$ \\
\hline
\end{tabular}

the threshold energy for the definite AR process, $\alpha$ is the process characteristic factor. At the calculations, we assume that above expressions in the Boltzmann approximation [2] can be also used for evaluations of the activation parameters for different AR processes at the degenerate conditions in electron and hole subbands in the QWs (Table 2) [13].

Initially, evaluations of the AR rate $R$ are carried out for each QW separately (Figs. 1 and 2) and then the obtained results are included in the determining of the lasing threshold versus the temperature.

Values of the current carrier concentration $n_{\text {th }}$ were varied from $1 \times 10^{13}$ to $3 \times 10^{13} \mathrm{~cm}^{-2}$ in the temperature range from 250 to $350 \mathrm{~K}$, assuming the conditions of the electroneutrality in the laser active region.

For the asymmetric QW heterostructure under consideration, the temperature dependence of the AR rate occurs to be activation-like. The effective energy of activation $E_{\text {act }}$ changes slightly with increasing the temperature and is affected by variations of $E_{\mathrm{q}}$. In parallel of the decreasing of the energy $E_{\mathrm{q}}$ at the temperature growth, the value of $E_{\mathrm{act}}$ in general decreases. The main contribution to the total AR rate is introduced by the CCCH and CLLS processes. Such a behaviour is associated with the lower specific $E_{\text {act }}$ that is caused with smaller values of $\delta_{\mathrm{m}}$ as compared to

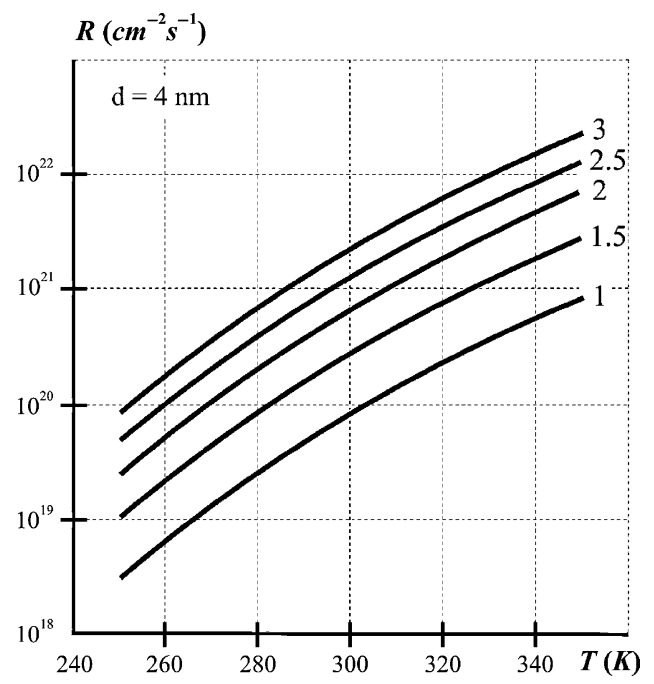

Fig. 1. Dependence of the total AR rate $R=C n_{\text {th }}^{3}$ on the temperature $T$ at different $n_{\mathrm{th}}$ (values in $10^{13} \mathrm{~cm}^{-2}$ at the curves), $d=4 \mathrm{~nm}$. 


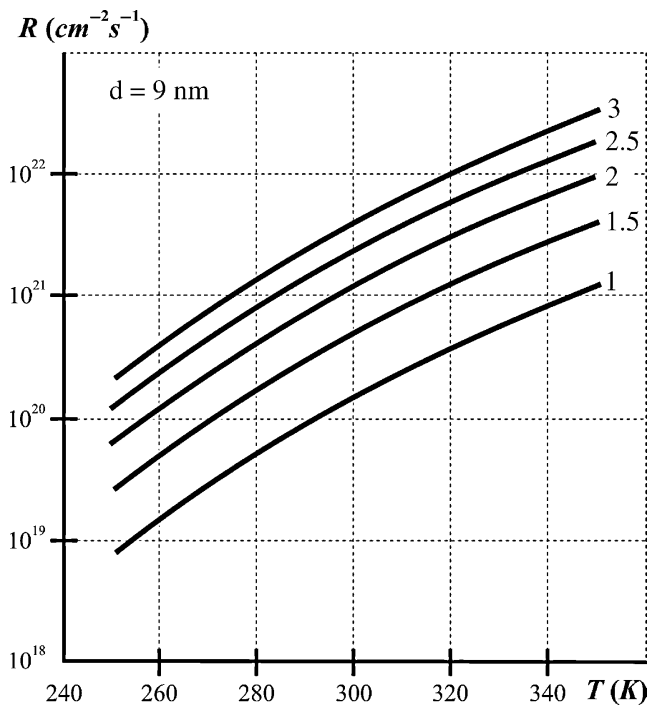

Fig. 2. Dependence of the total AR rate $R=C n_{\mathrm{th}}^{3}$ on the temperature $T$ at different $n_{\text {th }}$ (values in $10^{13} \mathrm{~cm}^{-2}$ at the curves), $d=9 \mathrm{~nm}$.

other examined AR processes. For the QWs with widths of 4-9 $\mathrm{nm}$ and for considered intervals of non-equilibrium carrier concentrations $n$ and temperatures $T$ the effective energy of activation $E_{\text {act }}$ lies in the range of $0.38-0.42 \mathrm{eV}$.

\section{Temperature dependencies analyzing}

The dependence $j_{\mathrm{th}}(T)$ is also markedly determined by the losses [11]. The dependence of lasing threshold via losses is determined with the coupling of the gain coefficient in the active area and $R_{\mathrm{sp}}$. Namely, the condition for the stable generation $\Gamma k(\nu)=k_{1}$, where $k(\nu)$ is the gain coefficient [11], and $R_{\mathrm{sp}}$ value which indicates the lasing beginning show such coupling. The coefficient of the linearity depends on the width $d$ and losses $k_{1}$. The threshold rate of the spontaneous radiative recombination in the quantum wells increases linearly with increasing the temperature as well [11]. Accordingly, the lasing threshold current density $j_{\text {th }}$ follows a similar law.

At the operation temperature $T=300 \mathrm{~K}$ and low losses $k_{1}=40 \mathrm{~cm}^{-1}$, the lasing threshold is about of $1.8 \mathrm{kA} / \mathrm{cm}^{2}$. At the larger losses and higher operation temperatures the lasing threshold grows up to $11 \mathrm{kA} / \mathrm{cm}^{2}$. Therewith, the threshold concentration of non-equilibrium current carriers in the QWs markedly increases. As a result, contribution of the AR rate in the lasing threshold has to enhance additionally the temperature dependence $j_{\mathrm{th}}(T)$ (Fig. 3) while the threshold current density resulting from only spontaneous radiative recombination increases linearly with increasing the temperature.

With increasing the operation temperature and losses in the cavity the quantum yield of spontaneous emission $\eta_{\mathrm{sp}}$ markedly drops (Fig. 4), where $T_{\mathrm{op}}=360 \mathrm{~K}$ and $k_{1}=$ $80 \mathrm{~cm}^{-1}$, the value of $\eta_{\mathrm{sp}}$ occurs to be of the order of 0.2 .

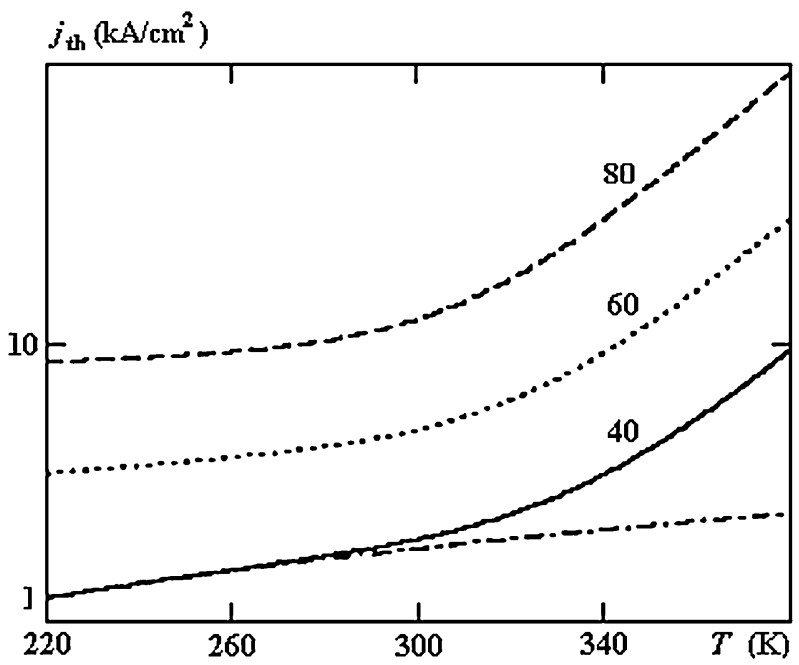

Fig. 3. Temperature dependence of the threshold current density $j_{\mathrm{th}}(T)$ at different losses $k_{1}$ (values at the curves in $\mathrm{cm}^{-1}$ ). The lower dot-and-dash curve corresponds to $k_{1}=40 \mathrm{~cm}^{-1}$ and the absence of AR processes in the laser active region.

In this case, taking into account the index $n=1$ and effective activation energy $E_{\text {act }} \approx 0.37 \mathrm{eV}$ one can evaluate according to relation (3) the value $T_{0} \approx 30 \mathrm{~K}$, i.e. the harmful decreasing of the characteristic temperature parameter of the threshold is revealed. It is clearly indicated in Fig. 3.

Comparison of the contribution of spontaneous radiative recombination and non-radiative AR processes in the lasing threshold indicates that the quantum yield of spontaneous emission $\eta_{\text {sp }}$ maintains sufficiently high at the threshold until the operation temperature top does not exceed $340 \mathrm{~K}$ and the cavity loss $k_{1}$ is lower than $60 \mathrm{~cm}^{-1}$. In this case, we obtain that $\eta_{\mathrm{sp}} \geq 0.7$. The suppressed role of the AR in the threshold results from the conditions that the basic contribution to the spontaneous radiative recombination provides by the wider QW but the gain is determined in general by the narrower QW.

Consequently, the restraining of AR in the investigated lasers can be realized by the presence of a high-quality cavity and operating at room temperatures.

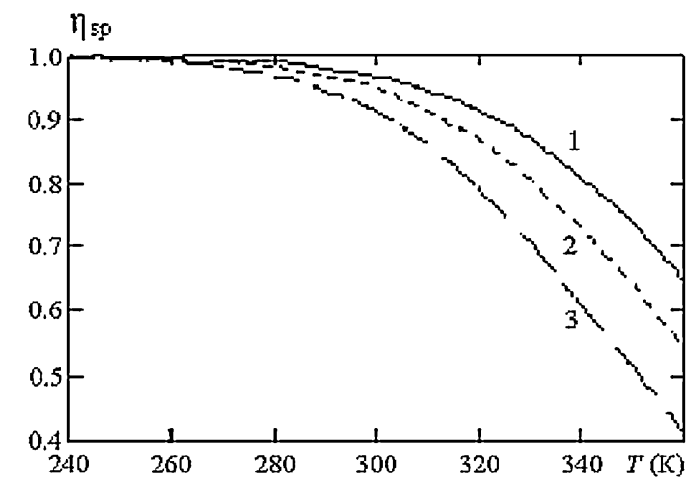

Fig. 4. Temperature change of the quantum yield of spontaneous recombination $\eta_{\mathrm{sp}}(T)$ at different losses (1) $k_{1}=40$, (2) 60, and (3) $80 \mathrm{~cm}^{-1}$ and attributed with the involved AR processes in the laser active region. 


\section{Conclusions}

We have presented a set of characteristics indicating the temperature behaviour of the lasing threshold for GaInAs-GaInAsP-InP AMQW heterolasers through the processes which affect such behaviour. For this, AR processes were firstly taken into consideration in calculations of AMQW lasers. Thus, AR rate changing in response to growth both an operating temperature and threshold carrier concentration has been shown and analysed. As it turned out, the most important contribution of the AR rate to the threshold of the emitted at the wavelength near $1.55 \mu \mathrm{m}$ is attributed with the $\mathrm{CCCH}$ and CLLS processes. Nevertheless, in the frameworks of carried out investigation, it has been shown that for the described asymmetric QW heterostructure lasers the restriction of AR processes on the temperature behaviour of the lasing threshold is not essential until the temperature of the active region is lower than $340 \mathrm{~K}$ and the cavity losses do not exceed $60 \mathrm{~cm}^{-1}$.

\section{References}

[1] B. Lee, C. Lin, IEEE Photon. Techn. Lett (1988) 322.

[2] G.P. Agrawal, N.K. Dutta, Long-Wavelength Semiconductor Lasers, Wiley, New York, 1986.

[3] S.R. Bank, M.A. Wistey, L.L. Goddard, et al., IEEE J. Quantum Electron. 40 (6) (2004) 656.

[4] V.K. Kononenko, A.A. Afonenko, I.S. Manak, S.V. Nalivko, OptoElectron. Rev. 3 (8) (2000) 241.

[5] A.A. Afonenko, I.S. Manak, V.A. Shevtsov, V.K. Kononenko, Semiconductors 31 (9) (1997) 929.

[6] V.K. Kononenko, V.I. Tsvirko, Bull. RAS, Ser. Phys. 67 (2) (2003) 223.

[7] E.P. O'Reilly, A.R. Adams, IEEE J. Quantum Electron. 30 (2) (1994) 366.

[8] P.J.A. Thijs, L.F. Tiemeijer, J.J.M. Binsma, T. Dongen, IEEE J. Quantum Electron. 30 (2) (1994) 477.

[9] V.K. Kononenko, D.V. Ushakov, I.A. Sukhoivanov, O.V. Mashoshina, Proc. LFNM'2004, Kharkiv, Ukraine (2004) 107.

[10] Z.-M. Li, T. Bradford, IEEE J. Quantum Electron. 31 (10) (1995) 1841.

[11] I.A. Sukhoivanov, O.V. Mashoshyna, V.K. Kononenko, D.V. Ushakov, Proc. SPIE 5582 (2003) 203.

[12] S.L. Chuang, Phys. Rev. B 43 (12) (1991) 9649.

[13] I.A. Sukhoivanov, O.V. Mashoshina, V.K. Kononenko, D.V. Ushakov, WSEAS Trans Math. 2 (3) (2004) 322-328. 